Supporting Information

\title{
Transparent molecular adhesive enabling mechanically stable ITO thin films
}

Shingyu Bok, Hae-Jun Seok, Yun Ah Kim, Jin-Hyeok Park, Jihyun Kim, Joohoon Kang, ${ }^{*}$ Han-Ki Kim,* and Byungkwon Lim*

School of Advanced Materials Science and Engineering, Sungkyunkwan University (SKKU), Suwon 16419, Republic of Korea

E-mail: joohoon@skku.edu (J. K.), hankikim@skku.edu (H.-K.K.), and blim@skku.edu (B. L.)
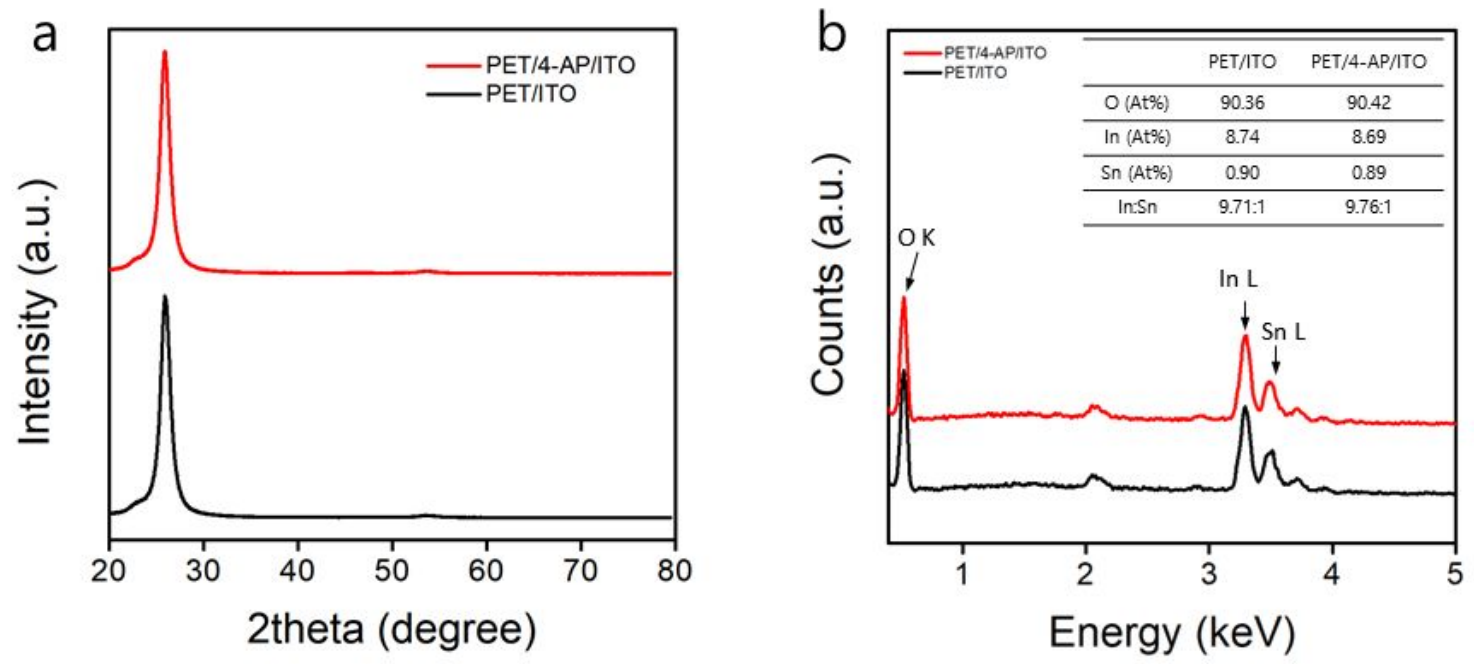

Figure S1. (a) X-ray diffraction patterns of PET/ITO and PET/4-AP/ITO electrodes (the strong peak around $26^{\circ}$ is originated from PET substrate. (b) Energy Dispersive X-ray Spectroscopy spectra of PET/ITO and PET/4-AP/ITO electrodes. 


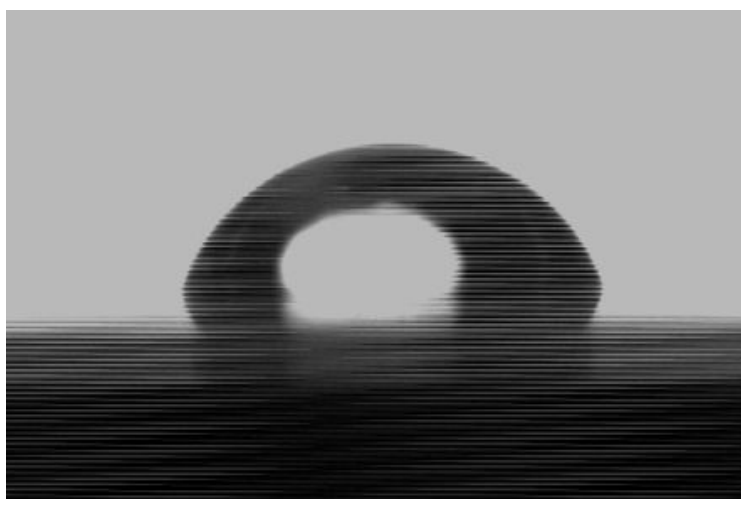

Bare PET $\left(68.4 \pm 0.4^{\circ}\right)$

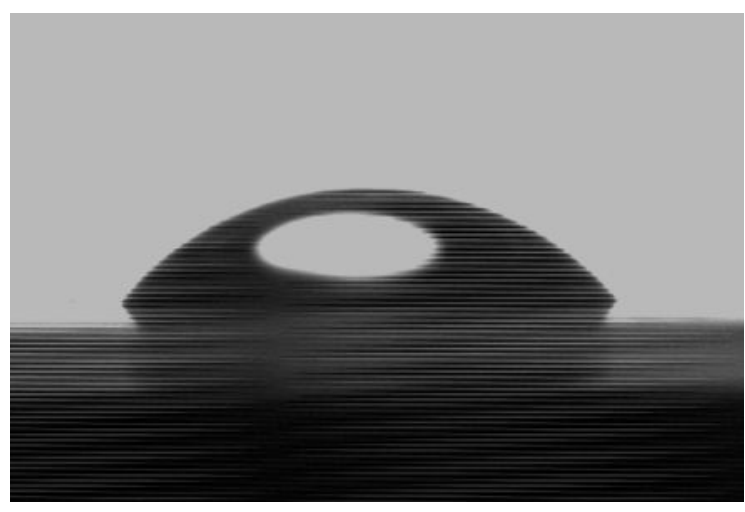

PET/4-AP $\left(48.8 \pm 1.2^{\circ}\right)$

Figure S2. Contact angles on PET and 4-AP/PET were $68.4^{\circ}$ and $48.8^{\circ}$, respectively. 

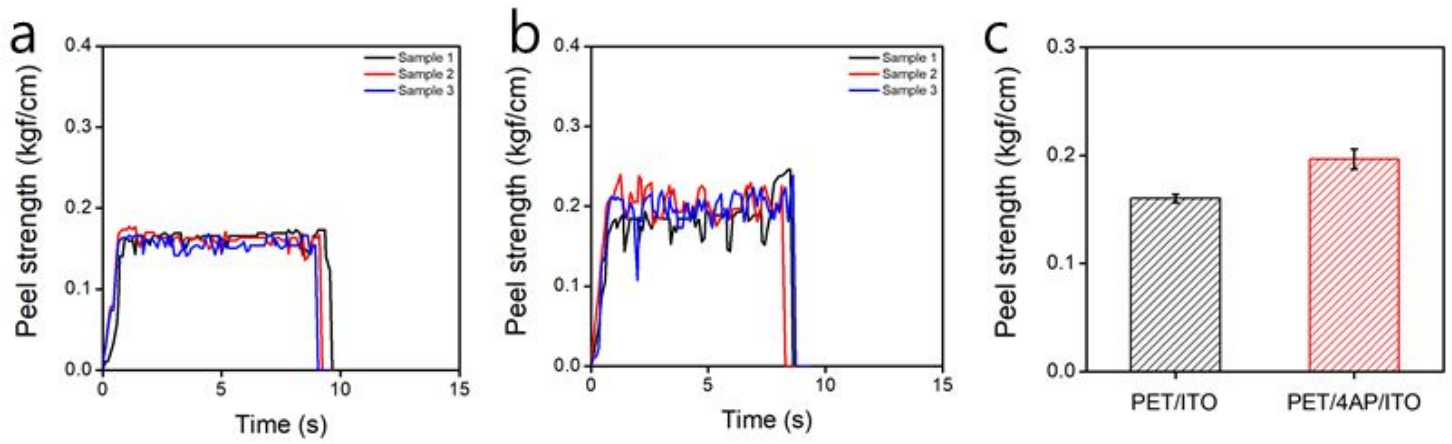

Figure S3. 180-degree peel test results of (a) PET/ITO and (b) PET/4-AP/ITO. (c) Average peel strength of PET/ITO and PET/4-AP/ITO. 

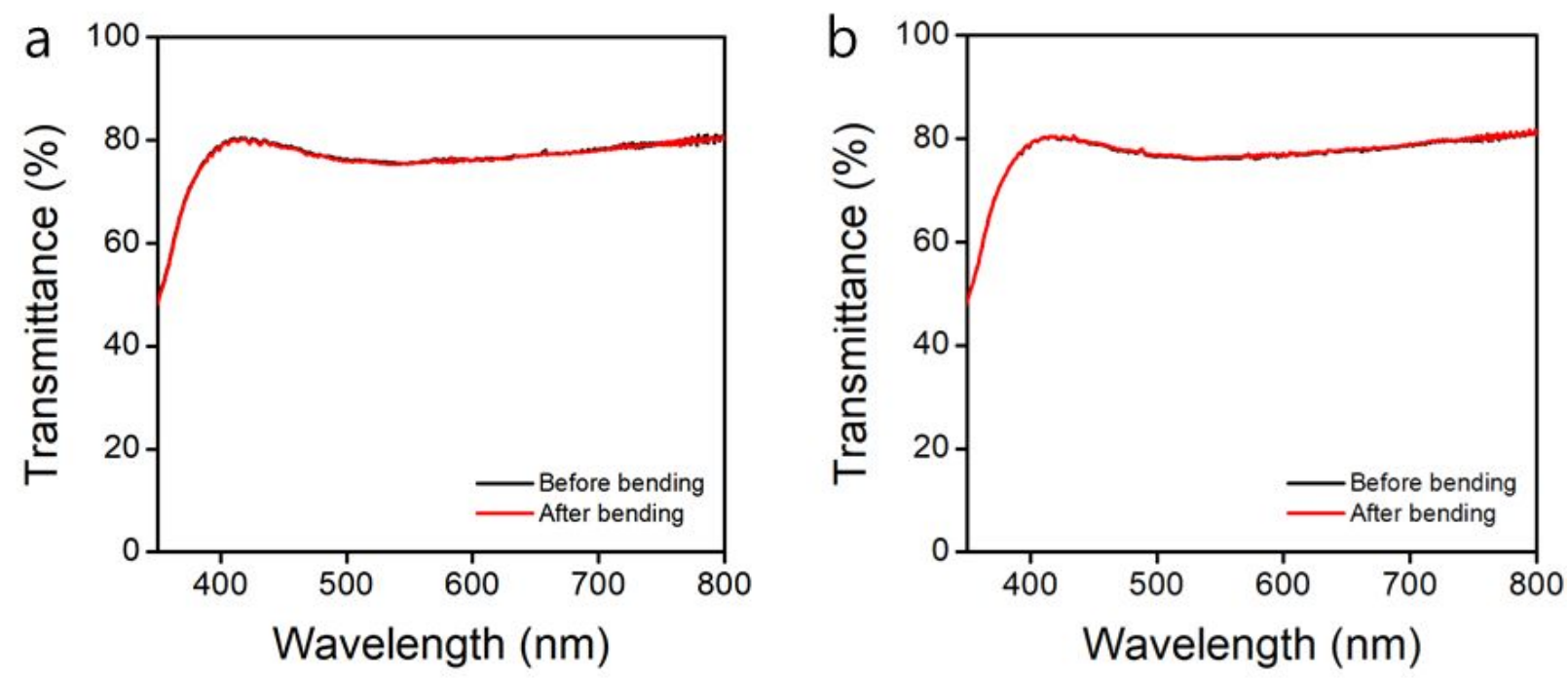

Figure S4. UV-vis spectra of (a) PET/ITO and (b) PET/4-AP/ITO electrodes before and after cyclic bending test. 


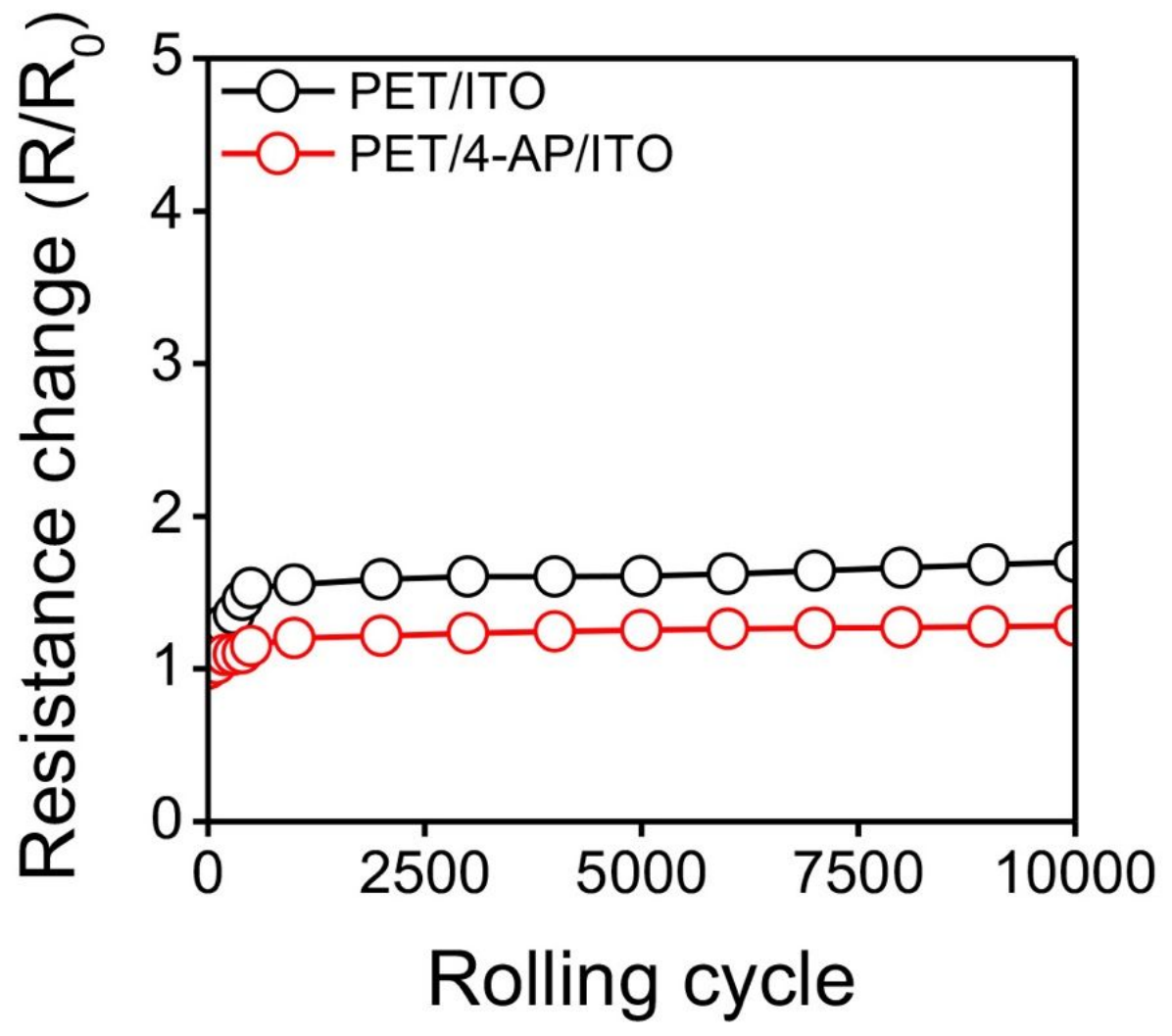

Figure S5. Resistance change with respect to the inner rolling cycle. 

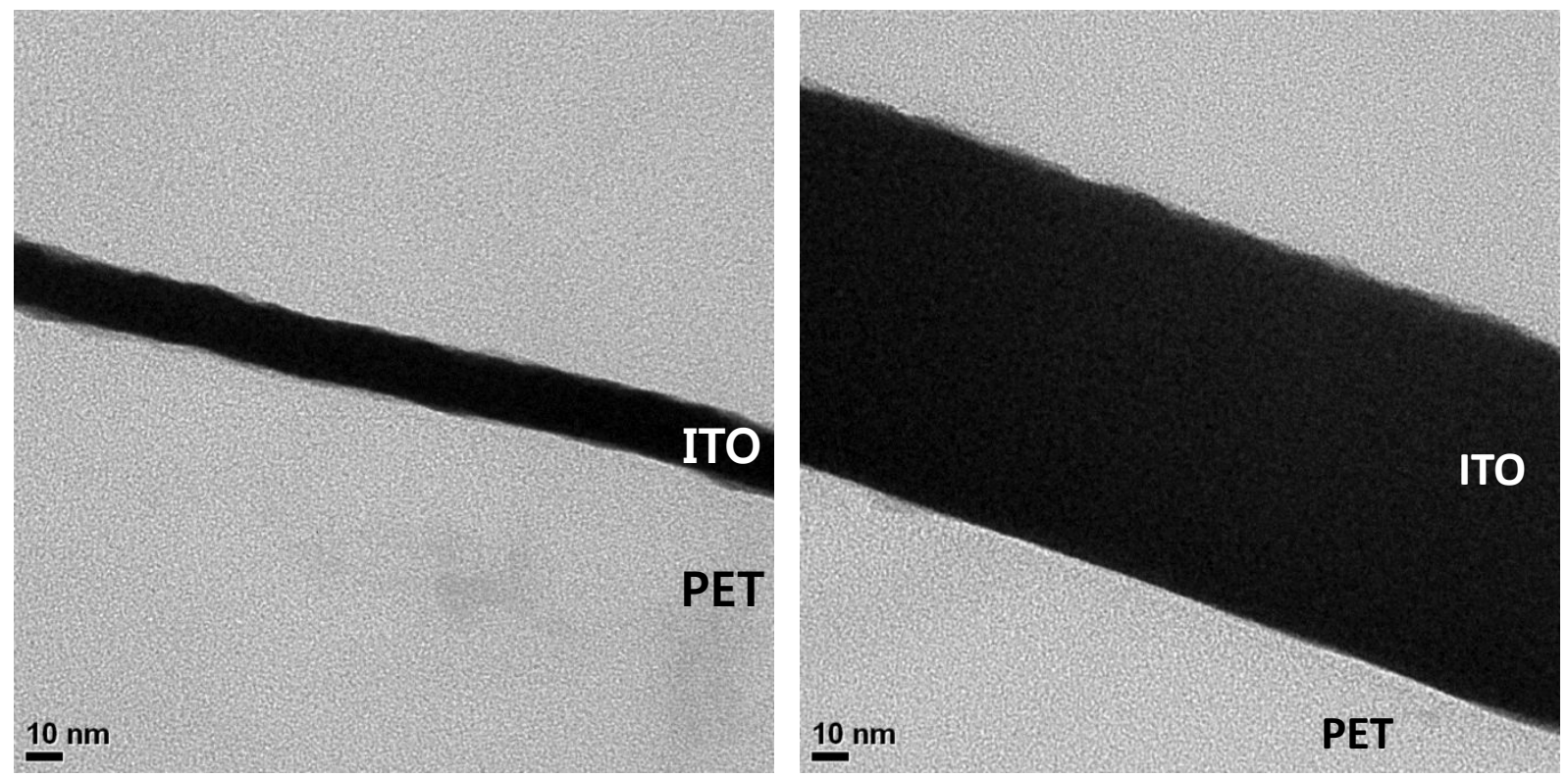

Figure S6. Cross-sectional TEM image of $20 \mathrm{~nm}$ (left) and 100-nm-thick (right) ITO on a PET substrate. 

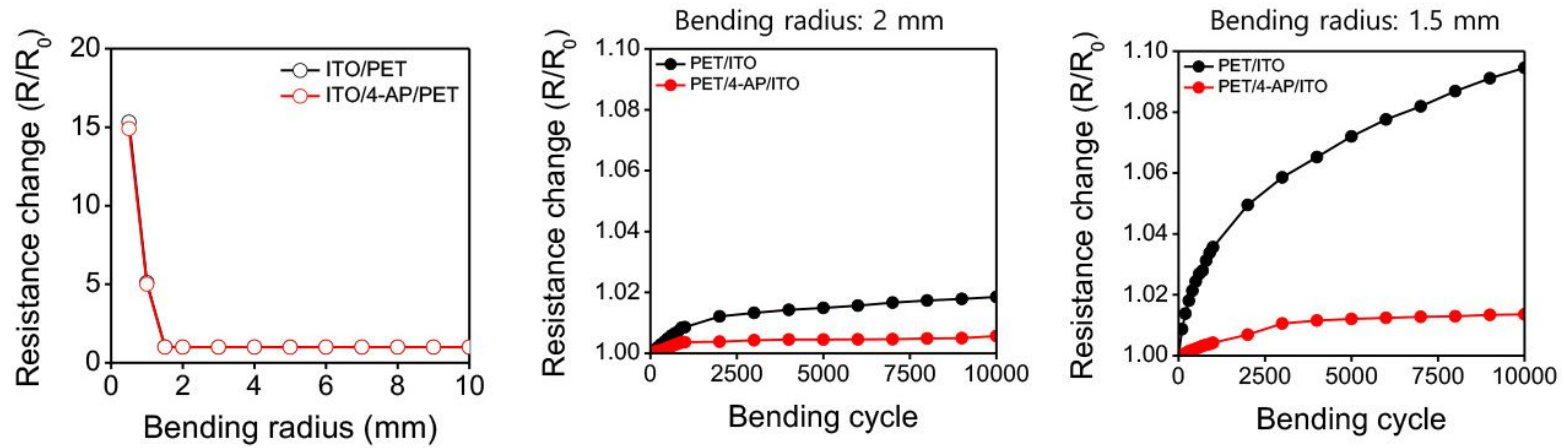

Figure S7. Resistance changes of the $20 \mathrm{~nm}$-thick ITO on the PET and 4-AP/PET as the bending radius was reduced. Resistance changed as cyclic bending motion was applied at a fixed bending radius of 2 and $1.5 \mathrm{~mm}$, respectively. 

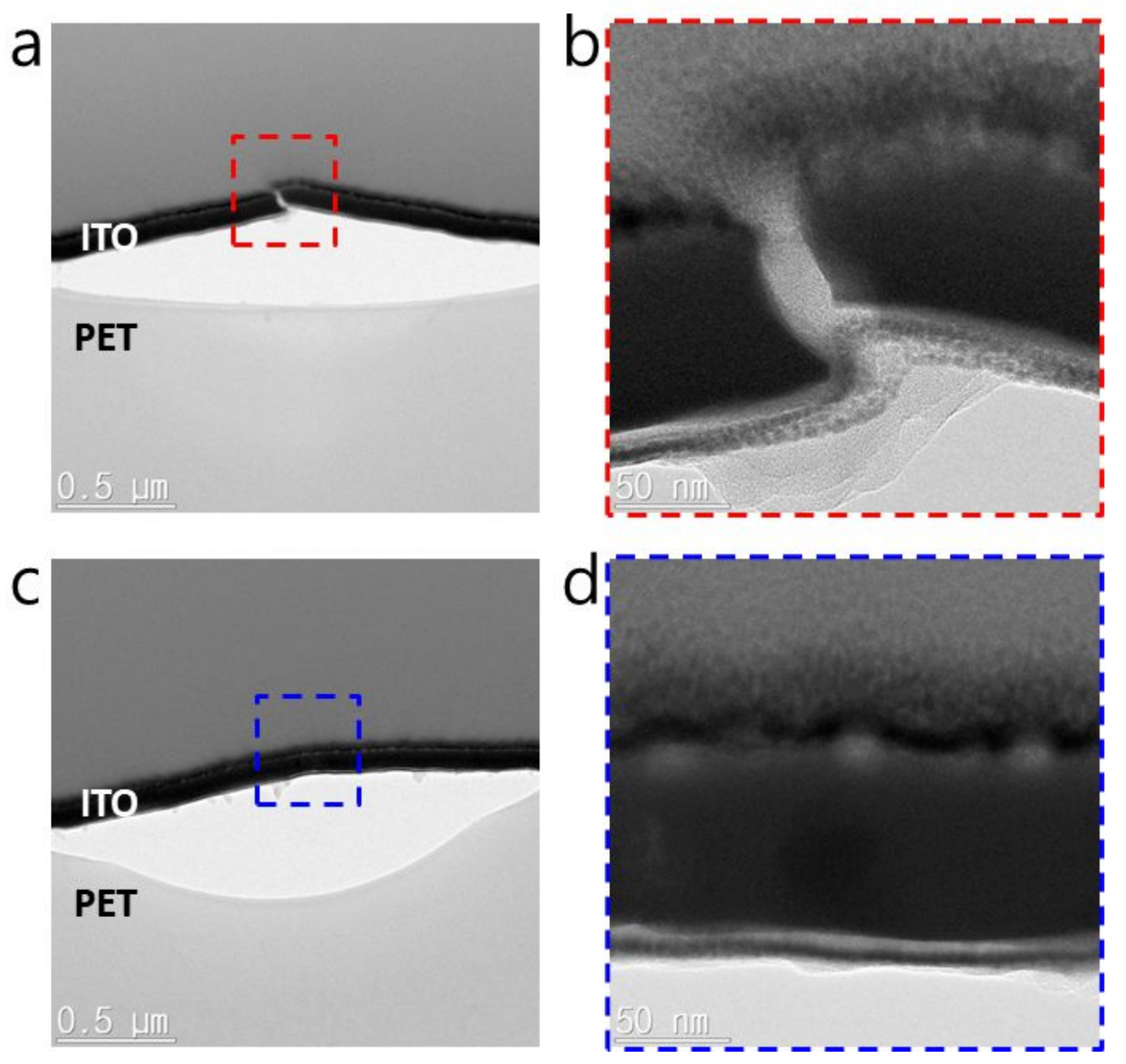

Figure S8. Cross-sectional TEM images of (a, b) PET/ITO and (c, d) PET/4-AP/ITO electrodes after 10,000 cycles of bending stability test at $8 \mathrm{~mm}$ of bending radius. 

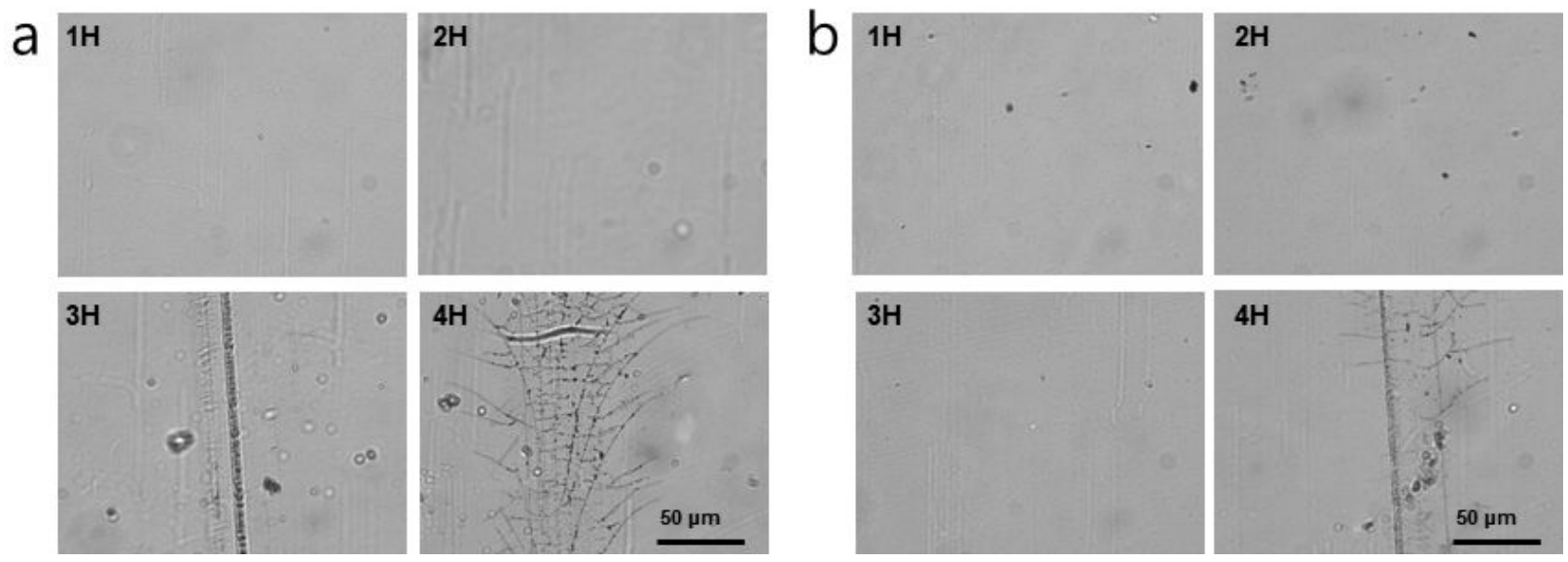

Figure S9. Optical images of the (a)PET/ITO and (b)PET/4-AP/ITO surfaces after pencil hardness test. 
Table S1. Bending stability of ITO electrodes with various adhesive layers

\begin{tabular}{ccccc}
\hline $\begin{array}{c}\text { Structure } \\
\text { (thickness) }\end{array}$ & $\begin{array}{c}\text { Bending radius } \\
\text { for cycle test }(\mathbf{m m})\end{array}$ & Bending cycles & $\begin{array}{c}\text { Resistance } \\
\text { change }\left(\mathbf{R} / \mathbf{R}_{\mathbf{0}}\right)\end{array}$ & ref \\
\hline $\begin{array}{c}\mathrm{PET} / 4-\mathrm{AP} / \mathrm{ITO} \\
(50 \mu \mathrm{m} / \sim 1 \mathrm{~nm} / 20 \mathrm{~nm})\end{array}$ & 1.5 & 10,000 & 1.01 & This \\
$\begin{array}{c}\mathrm{PET} / 4-\mathrm{AP} / \mathrm{ITO} \\
(125 \mu \mathrm{m} / \sim 1 \mathrm{~nm} / 100 \mathrm{~nm})\end{array}$ & 8 & 10,000 & 1.04 & work \\
\hline $\begin{array}{c}\mathrm{PET} / \mathrm{ZrCu} / \mathrm{ITO} \\
(125 \mu \mathrm{m} / 3 \mathrm{~nm} / 30 \mathrm{~nm})\end{array}$ & 16.5 & 10,000 & 1.4 & {$[1]$} \\
\hline $\begin{array}{c}\mathrm{PET} / \mathrm{AgMg} / \mathrm{ITO} \\
(50 \mu \mathrm{m} / 10 \mathrm{~nm} / 30 \mathrm{~nm})\end{array}$ & 7 & 10,000 & 1.42 & {$[2]$} \\
\hline $\begin{array}{c}\mathrm{PET} / \mathrm{AgZr} / \mathrm{ITO} \\
(50 \mu \mathrm{m} / 10 \mathrm{~nm} / 30 \mathrm{~nm})\end{array}$ & 7 & 10,000 & 1.49 & {$[3]$} \\
\hline $\begin{array}{c}\mathrm{PET} / \mathrm{CuMg} / \mathrm{ITO} \\
(50 \mu \mathrm{m} / 10 \mathrm{~nm} / 30 \mathrm{~nm})\end{array}$ & 7 & 10,000 & 1.21 & {$[4]$} \\
\hline $\begin{array}{c}\mathrm{PET} / \mathrm{graphene} / \mathrm{ITO} \\
(180 \mu \mathrm{m} / 0.33 \mathrm{~nm} / 60 \mathrm{~nm})\end{array}$ & 8 & 10,000 & 1.3 & {$[5]$} \\
\hline
\end{tabular}

\section{References}

1. Lin, H.K.; Chiu, S.M.; Cho, T.P., Improved Bending Fatigue Behavior of Flexible PET/ITO Film with Thin Metallic Glass Interlayer, Mater. Lett. 2013, 113, 182-185.

2. $\quad$ Lin, H.K.; Chen, H.A.; Huang, U.G.; Chang, C.M.; Huang, J.C., Effects of Mg Content and Thermal Treatments on Optoelectronic and Bending Properties of Transparent Conductive Indium Tin Oxide/AgMg Bi-Layer Film, Thin Solid Film 2020, 697, 137842.

3. Chang, C.M.; Lin, H.K.; Huang, U.G.; Hong, H.A.; Huang, J.C., Effects of Different Annealing Processes on Optoelectronic and Bending Fatigue Properties of AgZr and ITO/AgZr Thin Film Metallic Glass, Opt. Laser Eng. 2019, 115, 100-106.

4. Lin, H.K.; Hong, S.Z., Effects of Mg Content and Annealing Treatment on Optical and Electrical Properties of CuMg and ITO/CuMg Metallic Glass Films, J. Alloy Compd. 2018, 248, 248-252.

5. $\quad$ Lee, S. J.; Kim, Y.; Hwang, J.-Y.; Lee, J.-H.; Jung, S.; Park, H.; Cho, S.; Nahm, S.; Yang, W. S.; Kim, H.; Han, S. H., Flexible Indium-Tin Oxide Crystal on Plastic Substrates Supported by Graphene Monolayer, Sci. Rep. 2017, 7, 3131. 